\title{
WIRELESS VOIP IMPLEMENTATION USING ASTERISK PBX AND OPEN SOURCE SOFTPHONE
}

\author{
$1^{\text {st }}$ Euclid Narte Odjidja, $2^{\text {nd }}$ Salah Kabanda, $3^{\text {rd }}$ William Akotam Agangiba, $4^{\text {th }}$ Richard Kofi Annan, \\ \{salah.kabanda@uct.ac.za ${ }^{2}$,wakkotam@umat.edu.gh ${ }^{3}$,rkannan@umat.edu.gh ${ }^{4}$ \} \\ Computer Science and Engineering, University of Mines and Technology Tarkwa, Ghana ${ }^{1}$, Information Systems, University of Cape town \\ south Africa ${ }^{2}$, Computer Science and Engineering, University of Mines and Technology Tarkwa, Ghana ${ }^{3}$,
}

\begin{abstract}
This paper reechoes the need to use VOIP-based communication channels in order to reduce the heavy cost burden of communication in Sub Saharan Africa and other developing countries. We focus specifically on the context of the campus environment and implement IP PBX to cut down the cost of placing and receiving voice calls, instant messaging and voice mails with other colleagues connected to an asterisk server on WIFI-enabled networks. We are motivated by the ease and cost-effectiveness of setting up WIFI-enabled IP network infrastructure which in recent times could be extended to allow inter-institutional linkage to allow collaboration and research.
\end{abstract}

Keywords: VOIP, Communication, Mobile Data, Cost.

\section{Introduction}

Countries situated in Sub Saharan Africa and other developing countries in general, have traditionally been characterised as having poor physical infrastructure, such as roads and telecommunication. As a consequence, most countries in these regions have closed the chapter on obtaining telephone lines and fully embraced mobile communication. The existing evidence with regard to mobile communication show benefits in various facets of life such as in agriculture where improved market efficiency and reduced consumer prices for certain commodities has been reported [1], in health and trauma cases such as post-rape service delivery [2], and in the banking sector where mobile banking has brought on board the unbanked [3].

Although many benefits are reported of mobile communication for development, most users in Sub Saharan Africa continue to perceive high costs attached to the mobile internet communication emanating from the mobile data prices. As long as users continue to associate the internet with higher data prices, there will be no full maturity usage of the internet in Sub Saharan Africa, and more so users in low resource settings. For example, a survey of mobile phone usage in five African countries (Kenya, Uganda, South Africa, Ghana, and Nigeria) has found that Internet browsing via phones now stands at 40 per cent across these countries, with $51 \%$ of respondents in Ghana and $47 \%$ in Nigeria reporting that they use their phones to access the internet. South Africa lags behind at 40\%, and Kenya (34\%) and Uganda (29\%) are slowest on the uptake (http://www.itnewsafrica.com/). Consumers in South Africa, one of the country which has a higher increased uptake of smartphones, are at the forefront in voicing their discomfort with the high data prices via \#Datamustfall[4]. Comparatively, South Africa's data prices are much higher than those in other countries on the continent and in the world. This is concerning because according to [5], unless we tackle the issue of unaffordable internet, we're just going to go on entrenching and worsening the existing divides and inequalities in our country'. Consumers in the low remote areas of Sub Saharan Africa, who are unable to afford high costs of data, will be worse off in this digital age. This problem is more severe for students who are in areas with shortages of qualified teachers and traditional educational resources such as textbooks [6]. In some of these settings, studies have confirmed that access to the internet does contribute to student learning. For example, [7] report that students spent significantly more time using the university's learning management system when classes were in session. [8] Found that sixty percent of medical students in Pakistan used the internet sites to access subjects on medical topics. They report less utilization of internet however which was due to slow speed of data transfer. [9] Shows how effective mobile internet is in a learning system in secondary and higher secondary level students.

The context of this study is therefore in higher education institutions where students continue to face challenges of access to the internet and learning material. Students have mobile phones which can aid their learning for example having a chat discussion about a specific topic, but this is hampered by mobile data costs. When students attempt to make communication with fellow students on matter related to academia, they incur high costs because even though they reside in the same campus, the call signaling and voice traffic are always routed to the core network, which incurs extra telephony cost [10]. This study proposes a test-base using Zoiper (SIP clients) and asterisk server Private Branch Exchange (PBX) for an intranet VoIP application. The Zoiper and asterisk intranet VoIP implementation allows free communication without the need for telecommunication provider charges be it call or data rates [11]. Zoiper (SIP client) is based on a custom configured PBX using the 
asterisk server to facilitate student communications across multiple university campus setting in developing countries. [12] defined PBX as a telephone system that switches calls between users within a small area, such as a hotel, an office building, or a hospital. Most higher institutions of learning have installed wired and wireless intranet (e.g. LAN) making it possible for them to implement and manage their own PBX. The campus PBX using Zoiper and asterisk is therefore intended to tap into these intranet services to allow students easier and free communication, specifically make phone calls and have instant messaging services. Students can make phone calls and have instant messaging services. Although PBX is an old technology, it has not yet been fully utilized in the education context of Sub Saharan Africa and this study considers PBX a paramount solutions for the challenges of students in developing countries, and as [13] notes, PBX should be considered as part of a nation's critical infrastructure, along with the public telephone network

The rest of the paper is structured as follows: related works on PBX and its use in developing countries is covered in Section II. Section III outlines the research methodology. Section IV presents the findings. Section V discusses the findings and section VI concludes the paper.

\section{Related work}

\subsection{Mobile data costs in developing countries}

For internet utilization to spread and become mature in Sub Saharan Africa, mobile data prices have to be perceived affordable or free for the majority of people, both for the urban and rural communities. According to Tariffic (FinTech.com), some countries in Africa, such as South Africa, face higher data costs when compared to BRICS-member countries Brazil, Russia, India and China, and that 'data prices for South Africa were on average $134 \%$ more expensive than the cheapest prices in the group". South African consumers have publicly made the awareness of high data costs and have called for its reduction through various channels such as the \#DataMustFall [4] initiative [14] [4].

As more countries start to adopt smart phones and the need for mobile data increases, cost will remain a problem. According to the latest study by [15] the common use of mobile phones in Africa includes sending text messages, taking pictures or video, and mobile banking. Other reasons include getting political news, accessing a social networking site, getting health and consumer information and looking for a jobs. Even in countries with low data costs in Sub Saharan Africa such as Tanzanian, there remains low mobile internet usage. According to [16], one of the principle reasons, specifically amongst users situated in low resource settings (rural areas, example farmers) is because "of price charged per MB when downloading info/docs or browsing websites e.g. Google". These findings show the significant importance of findings solutions to address data costs in developing countries where, average monthly fixed broadband prices are three times higher than in developed countries and mobile broadband prices are twice as expensive as in developed countries [17] To attain internet usage maturity, there is need to decrease the prices because the decrease in mobile-broadband prices goes hand in hand with an increase in the intensity of use [17].

\subsection{PBX application use in the context of higher institutions}

Although the use of the internet in developing countries is low, this could potentially change if internet use is perceived affordable and if possible free, especially in areas perceived to be either critical for operations such as in health or important for supplementing existing educational material. Those in the education sector have since identified the importance of ICT and the internet in particular in learning for students who reside in areas where access to learning material and teachers is a challenge. Most learning institutions in developing countries have now access to Internet but its use for most students remains a challenge due to the high cost. As a consequence, students remain at a disadvantage when comparing them to students in the developed economies.

Several solutions have been proposed to address student access to the internet within their limited local such as a campus, but few focus on the context of Sub Saharan Africa. One example is the VillageCell solution by [18] of multiple base stations which are connected through the local wireless network, and calls are routed via private branch exchange (PBX) servers implemented in an open source framework (Asterisk). VillageCell in Africa allows free calls within the local network and standard connections to outside callers using the satellite link. It also uses free, open source solutions and off-the-shelf hardware, and hence the total deployment cost is minimal and the solution is scalable.

Examples that feature specifically in the context of education campus include the works of [19] who designed and implemented an Asterisk server to serve as the local exchange for placing voice and video calls within a private Wi-Fi cloud and legacy networks (Public Switch Telephony Network). Their model which is accessible within the area of a university campus, allows only those mobile phones and PCs' to connect to the Asterisk server which are registered with the wireless network. [20] Developed a voip based asterisk pbx on ubuntu 11.10 which allow unlimited free calls with the help of ip phone or traditional phone within a lan/can without internet connection. For wireless communication, the authors whose study was situated in Asian context where there is limited bandwidth, extended voip based pbx to wi-fi technology. [21] Present a VoIP based solution designed for the use over a campus environment whose operating costs of phone systems take a large part of the budget. Their 
proposed solution is designed to carry all telephony signals by offering a new communication tool in which data packet streams are transmitted over IP. Like a standard SIP server, the solution is a IP software which can simultaneously act as a phone gateway for the phone system.

\section{Methodology}

VoIP technique is gradually becoming the most common communication system as a result of its benefits over the Public Switched Telephone Network (PSTN). This communication technique is popular to many who are not new to using softphone application such as Skype, WhatsApp, Imo, Facebook and the likes. VoIP offers less expenditure as well superior flexibility, management and support compared to the PSTN [22]. Hence, for this study, VoIP implementation using campus IP intranet was used to explore the most economical means of communication and exchanging information. Not only can campus VoIP deliver voice services, but stakeholders could appreciate multimedia sessions such as video conferencing, telepresence, instant messaging and fax data over intranet using Internet Protocol at no usage cost. In this study we propose a test-based Wi-Fi-enabled campus intranet with VoIP integration using Zoiper softphone (SIP clients) and asterisk server as the Internet Protocol (IP)-PBX. The PBX is a private telephone exchange allowing multiple telephones to be connected together for communication [19].

\subsection{System Architecture}

Three VoIP software packages, Asterisk, Trixbox, and PBX in a Flash (PBXiF), were selected for testing as a replacement for the old PBX system. These selections were made based on three criteria: 1) cost of acquisition and support, 2) availability of forums or technical support groups, and 3) compatibility with industry standard hardware platforms [23]. The most known telephone software for processing calls and providing a powerful control over call activity is Asterisk [24] therefore; this study used the Asterisk IP PBX, a system that enables call routing and other VoIP functions for the VoIP phones [24]. PBX is situated on the network Infrastructure layer which is responsible for communications where the Delivering information and transferring Voice are based on specific protocols, such as session initiation protocol (SIP) and real time protocol (RTP) [25].

The SIP client softphone (Zoiper) was opted for the campus intranet VoIP solution as it the SIP is among one of the widely used voice signalling protocols developed by the Internet Engineering Task Force -IETF [26]. Furthermore, [22] mentions SIP forms the major basis as the standard signaling for IP networks to enable VoIP. Thus, PBX and network systems that do no support SIP would be replaced with SIP enabled PBX and network systems that packet switched and IP enabled. Figure 1 shows the IP PBX and its connections to the various client softphones; the sip:6003@192.168.43.82 and sip:6004@192.168.43.82 SIP addresses are used by the IP PBX to identify and communicate with the various SIP-client softphones on the campus intranet.

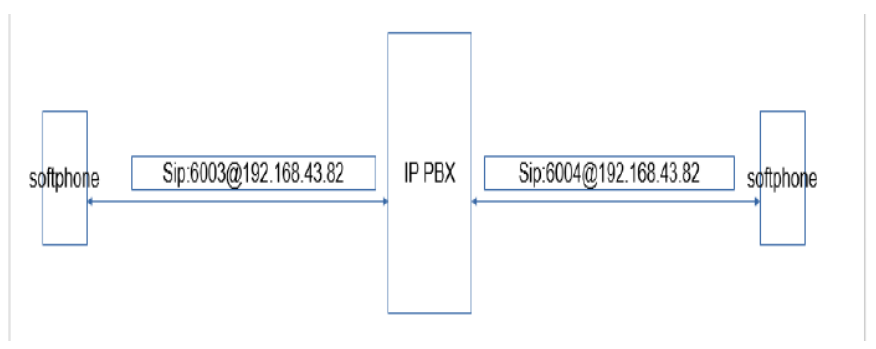

Fig.1. The IP PBX SIP registration Diagram

With this setup, the operational cost is significantly reduced, since the voice communication infrastructure becomes part of the campus intranet, instead of being provided Telephone Company. Another important consideration, is that we use an open source PBX called Asterisk to develop this layer. Furthermore, to check the user's connection (in telephony this requirement is called presence), we set up an openfire server connected to the PBX to manage presence feature. We used Asterisk as it is an open source PBX and it supports all the security techniques. The Asterisk PBX software was loaded on two Dell Desktop machines [25].

\subsection{Implementation}

The Asterisk PBX softswitch is configured to use the SIP protocol for the establishment and termination of calls and SIMPLE for instant messaging. The Opus audio codec, which is supported by a large number of devices and software, is used for voice call support in the Asterisk PBX. It provides good voice quality even on lowbandwidth networks. Wireless access points are installed to allow SIP client softphones (Zoiper) communicate to each other within the IP intranet as indicated in Figure 2. The IP PBX is connected to a Wi-Fi router which takes 
the VoIP packets, decodes them, then re-encodes them into a wireless radio wave signal and broadcasts it into the air. Wi-Fi enabled SIP client handheld phone picks up the signal and receives the call.

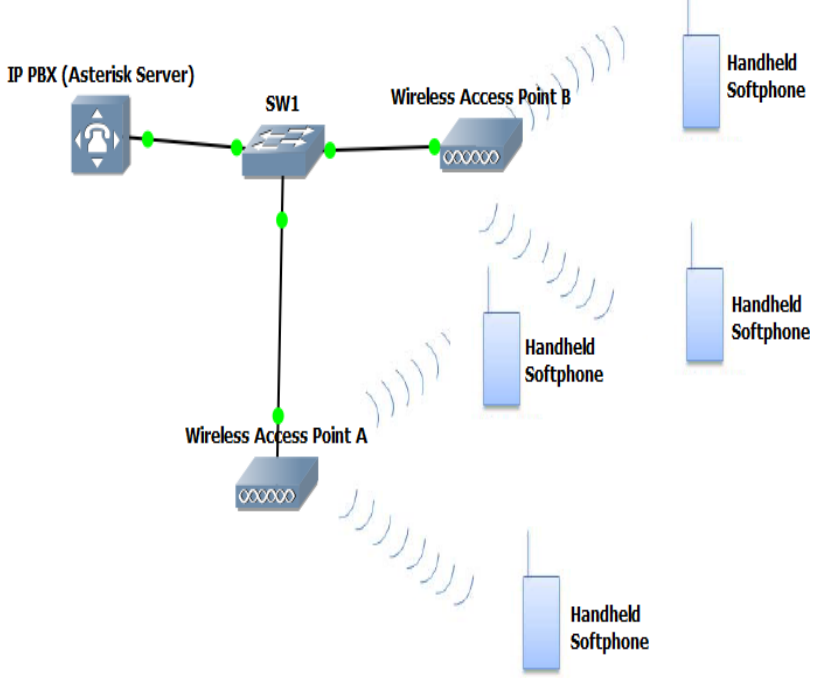

Fig.2. Basic Intranet Connection for PBX System

Calls are made from one SIP client to another through the Wi-Fi supported Zoiper android mobile phone application. The SIP client configuration is shown in Figure 3.

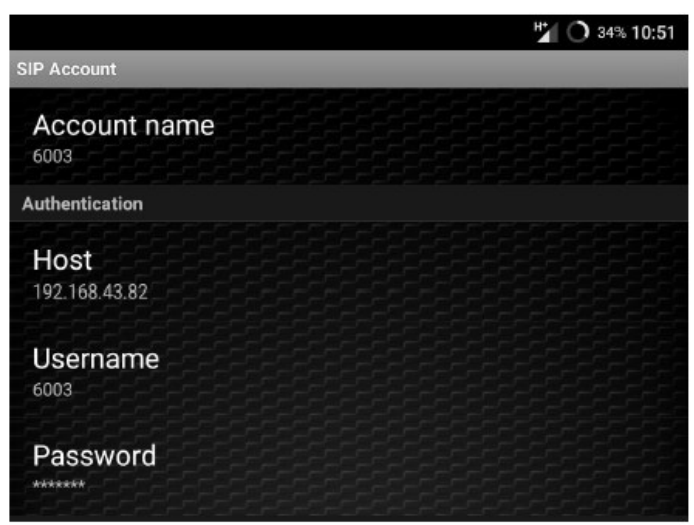

Fig.3. SIP-client Softphone Configuration

Following the SIP client configuration, was the creation of the dial plan which defines how asterisk handles the incoming and outgoing calls. The dial plan logic allows us to call between the extensions. The dial plan logical code is specified in the extensions.conf file in asterisk system to authenticate the callers and to provide different services such as voice mail, instant messaging etc. Figure $\mathbf{4}$ shows the dial plan designed for the system.

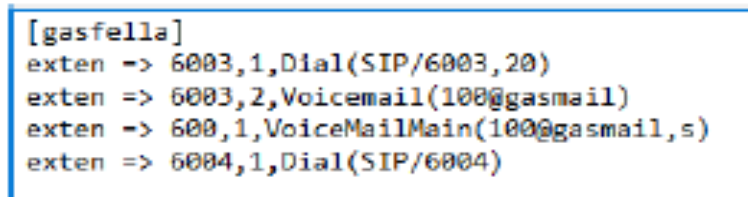

Fig.4. Dial Plan (IP Asterisk PBX)

A messaging context, gasms, has been added to facilitate instant messaging. Most importantly a message sent is retained and not lost in the system i.e. if the recipient is offline. It is automatically re-sent when the recipient comes back online. Hence, no need for the sender to reconstruct and re-send the message. The dial plan also facilitates voicemail functionality. Figure 5 shows the online and offline message configuration. 


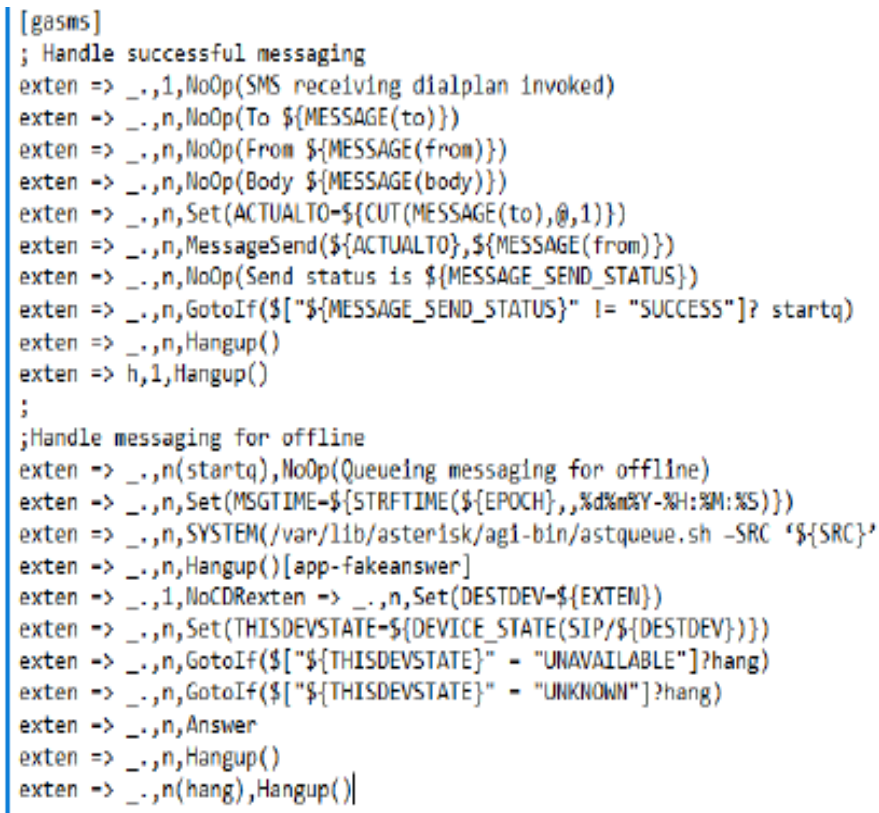

Fig.5. Online and Offline Messaging set-up

In order for clients to communicate with each other, accounts are configured for each phone in the channel driver which corresponds to the SIP protocol. The SIP channel driver used is chan_sip which is configured using the sip.conf file. Extensions are given to every client of the system to uniquely identify each user and are referenced by the dial plan to route calls to the right destinations. Figure 6 shows the configuration for the chan_sip channel driver.

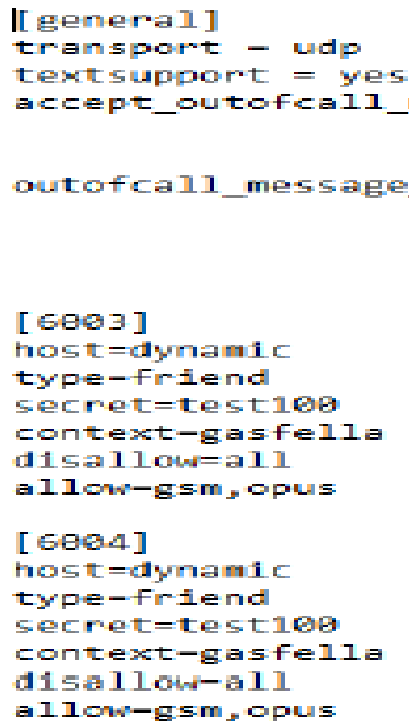

Fig.6. Chan_sip Channel Driver Configuration

\section{Findings}

With regards to testing the Asterisk PBX call process (refer to Figure 7), the SIP clients, being soft phones, register once with the Asterisk PBX server, and when they wish to make a call, they ask the PBX to establish the connection. When the connection is established, a channel brings the call to the Asterisk PBX, converting the analogue signal to a digital format by means of codecs (using the pulse code modulation technique (PCM)) and translated over the network. Each call is received and placed on a separate channel. 


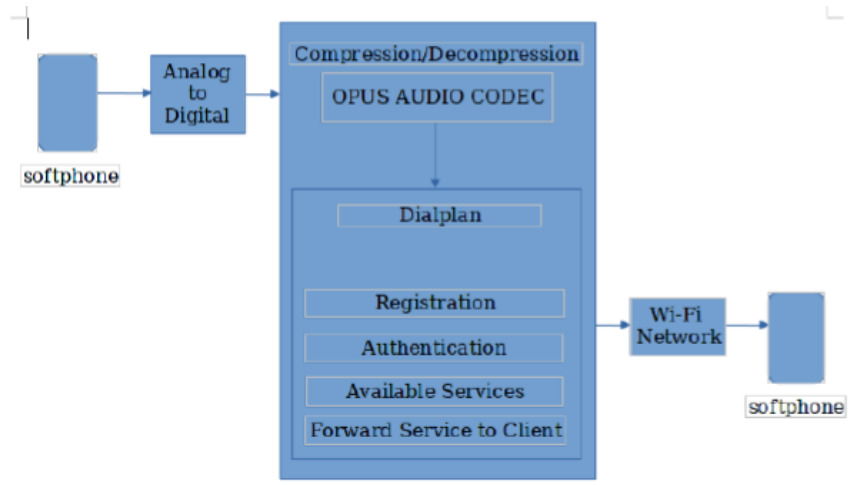

Fig.7. The Asterisk PBX call process.

The Asterisk PBX has a directory of all users and their corresponding SIP address and thus is able to connect a call to the desired destination. For example, Figure 8 shows one of the successful registrations of SIP clients 6003 and 6004 on the PBX system for call process.

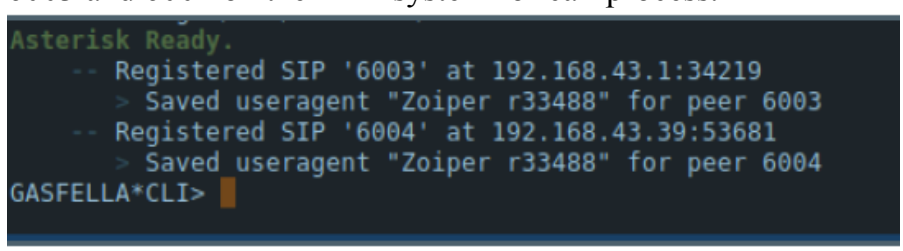

Fig.8. Successful Client Registration

The call processing takes place with the establishment of calls between successfully registered SIP clients and terminates afterwards. Figure 9 shows the communication establishment and termination between the registered SIP clients "6003" and "6004); whereas Figure 10 shows the instant messaging between registered clients.

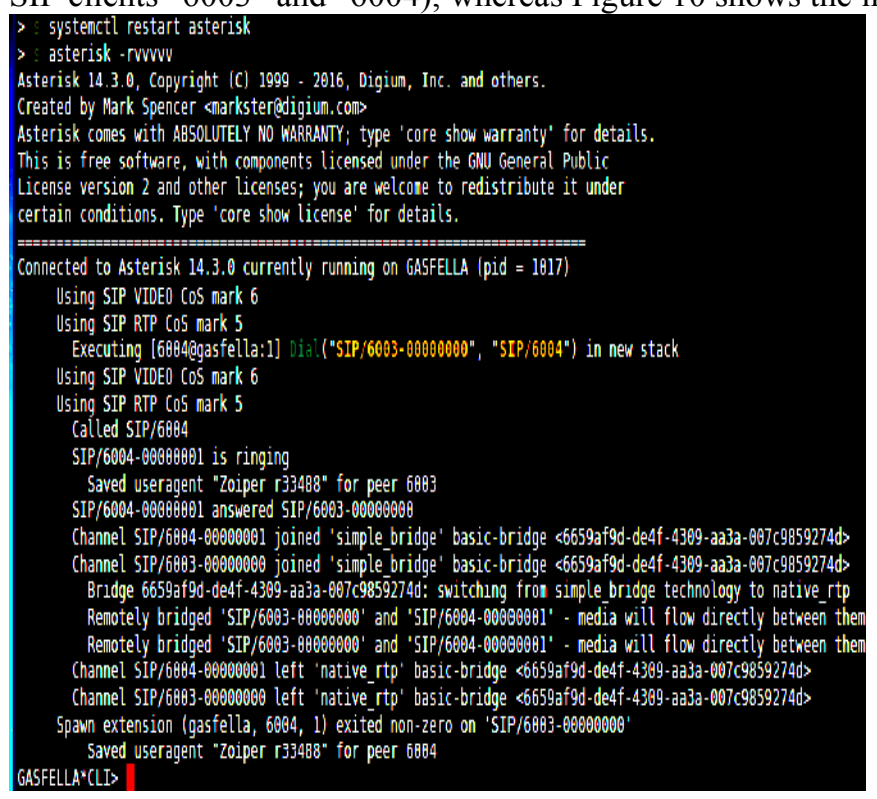

Fig.9. Successful Call Establishment and Termination. 


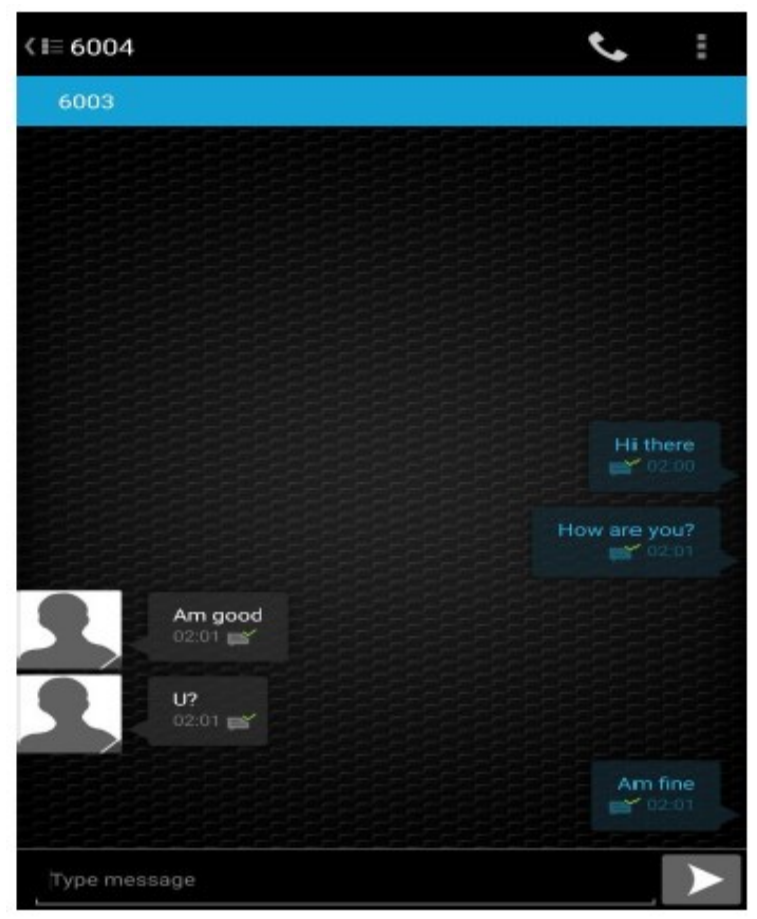

Fig.10. Instant Messaging Session between Clients

\section{Discussion}

Most developing countries and more specifically areas that are in remote areas tend to face challenges of communication, despite having access to a mobile phone. Usually, those who want to access the Internet are faced with the data cost problems which deter them from communication and ultimately internet use. This is a challenge more experienced by students in rural settings whose need for the internet resources is important to supplement their scarce resources they have such as books and adequate teachers. In this study, we address the problem of data cost by taking advantage of and implementing the IP PBX, which then addresses the communication needs of the already over-burdened student. Registered clients within the proximal range of the campus WIFI are able to communicate effectively with each other through calls, voice mails and instant messaging without having to incur any cost. Beyond the campus WIFI range, it is still possible to connect to the server through the internet in order to communicate with those who are also connected to the server either through the WIFI or the internet. Hence, some cost is incurred only in the case where the student is either beyond the campus wifi range or is communicating with a person not on the WIFI. The study therefore confirms earlier works on the economics of IP-PBX which makes Voice over IP (VoIP) a cost-effective alternative to the legacy PBX or key system [27].

\section{Conclusion}

The purpose of this study was to address the challenges experienced in developing countries around data costs which in turn hampers communication. Taking a higher education context as the setting for the study, we proposes a test-base using Zoiper (SIP clients) and asterisk server Private Branch Exchange (PBX) for an intranet VoIP application as a way of addressing the challenges students experience at higher institutions. The findings show that such a solution is effective and economical for institutions in developing countries. This study therefore is a contribution towards the ICT4D agenda of having existing or innovative ICTs address challenges experienced by people in developing country context.

\section{References}

[1] Aker, J. C., \& Fafchamps, M. (2015). Mobile phone coverage and producer markets: Evidence from West Africa. World Bank Economic Review, 29(2), 262-292.

[2] Mahlalela, N. B., Johnson, S., \& Mills, E. (2017). Feasibility and acceptability of a mobile phone intervention to improve post-rape service delivery in South Africa.

[3] Asongu, S. A. (2013). How has mobile phone penetration stimulated financial development in Africa?. Journal of African Business, 14(1), 7-18.

[4] \#DataMustFall. Available from https://www.facebook.com/datamustfall/ 
[5] Mann D (2016). Data Prices Are Too Expensive For Most. You Should Care. Available from http://www.huffingtonpost.co.za/dillon-mann/data-prices-are-too-expensive-for-most-you-should-care/

[6] Nye, B. D. (2015). Intelligent tutoring systems by and for the developing world: a review of trends and approaches for educational technology in a global context. International Journal of Artificial Intelligence in Education, 25(2), 177-203.

[7] Junco, R. (2014). iSpy: seeing what students really do online. Learning, Media and Technology, 39(1), 75-89.

[8] Ayatollahi, A., Ayatollahi, J., Ayatollahi, F., Ayatollahi, R., \& Shahcheraghi, S. H. (2014). Computer and Internet use among Undergraduate Medical Students in Iran. Pakistan journal of medical sciences, 30(5), 1054.

[9] Hassan, N., Ashraf, M. M., Abdullah, A., \& Murad, M. W. (2015). Mobile internet as a learning assistant for secondary and higher secondary students: the case of Bangladesh. In Australasian Conference on Business and Social Sciences (pp. 1296-1309).

[10] Lin, Y. B., Gan, C. H., \& Liang, C. F. (2010). Reducing call routing cost for femtocells. IEEE Transactions on Wireless Communications, 9(7), 2302-2309.

[11] Vennila, G., Shalini, S. N. and Manikandan, M. (2014) "Performance analysis of VoIP spoofing attacks using classification algorithms," 2014 Applications and Innovations in Mobile Computing (AIMoC), Kolkata, pp. 193198.

[12] K. Lee, K.C. Kang, and J. Lee (2002). Concepts and Guidelines of Feature Modeling for Product Line Software Engineering

[13] Androulidakis, I., Huerta, S., Vlachos, V., \& Santos, I. (2016, May). Epidemic model for malware targeting telephony networks. In Telecommunications (ICT), 2016 23rd International Conference on (pp. 1-5). IEEE.

[14] R. Serumula (2016). \#DataMustFall: Cut costs or else, Icasa warns. Available from http://www.iol.co.za/news/politics/datamustfall-cut-costs-or-else-icasa-warns-2072293

[15] Pew Research Center 2015. Cell Phones in Africa: Communication Lifeline Texting Most Common Activity, but Mobile Money Popular in Several Countries. Available from http://www.pewglobal.org/files/2015/04/PewResearch-Center-Africa-Cell-Phone-Report-FINAL-April-15-2015.pdf

[16] Furuholt, B., \& Matotay, E. (2011). The developmental contribution from mobile phones across the agricultural value chain in rural Africa. The Electronic Journal of Information Systems in Developing Countries, 48.

[17] ITU (2015) ICTFacts \& Figures. Available from https://www.itu.int/en/ITUD/Statistics/Documents/facts/ICTFactsFigures2015.pdf

[18] S. Nandi, S.Thota, Avishek Nag, Sw. Divyasukhananda, Partha Goswami, Ashwin Aravindakshan, Raymond Rodriguez, and Biswanath Mukherjee. (2016). Computing for Rural Empowerment: Enabled by Last-Mile Telecommunications

[19] Qadeer, M. A., Shah, K., \& Goel, U. (2012, May). Voice-video communication on mobile phones and PCs' using asterisk EPBX. In Communication Systems and Network Technologies (CSNT), 2012 International Conference on (pp. 534-538). IEEE.

[20] Shafi and Kawser (2015). Configuration of Own PBX System within a Campus Area Network and Implementation of VoWi-Fi. International Journal of Future Computer and Communication, Vol. 4, No. 6, December 2015

[21] Wei, X., Bouslimani, Y., \& Sellal, K. (2012, April). VoIP based solution for the use over a campus environment. In Electrical \& Computer Engineering (CCECE), 2012 25th IEEE Canadian Conference on (pp. 1-5). IEEE.

[22] Rattal, S., Badri, A., \& Moughit, M. (2014). A New Wireless VoIP Signaling Device Supporting SIP and H.323 Protocols. Journal Of Computer Networks And Communications, 2014, 1-7. http://dx.doi.org/10.1155/2014/605274

[23] R. Roysden and S.Z. Schiller, 2015. Retooling for success: a case study of voip implementation to improve customer service at a midwestern financial services office. Journal of the Midwest Association for Information Systems

[24] Hussain, M., Gupta, P., Bano, S., Kulkarni, V., Perigo, L., Williams, D., \& Engineer, F. (2016). High-Performance and Cost-Effective VoIP Security Techniques for Operations on IPv4, IPv6, and IPv4/IPv6 Networks.

[25] Vandenbosch and Nicolett 2016. An industry case study: a mobile-based business strategy to improve the customer care service in a major retail company

[26] Samsami, F., \& Akbari, A. (2013). Evaluation of Media Server in SIP-Based Voice Conferencing. International Journal Of Computer Theory And Engineering, 745-749. http://dx.doi.org/10.7763/ijcte.2013.v5.788

[27] Yu, J. (2016). An Empirical Study of Denial of Service (DoS) against VoIP. In Ubiquitous Computing and Communications and 2016 International Symposium on Cyberspace and Security (IUCC-CSS), International Conference on (pp. 54-60). IEEE. 
